\title{
De-blurring System for Dynamic Photography on a Linear Actuator
}

\author{
Chu-Hui Lee ${ }^{+}$and Zhi-Yong Qiu \\ Information Management Department, Chaoyang University of Technology, Taiwan
}

\begin{abstract}
In recent years, imaging technology has been flourishing innovation. There have developed a number of multimedia imaging equipment, such as vehicle driving recorders, smartphone photography, sport cameras, SLR cameras etc. Although the technique in these multimedia imaging equipment reaches a certain level, but there are still some potential issues of the image analysis, such as improving the quality of the motion blurred image. These potential challenges are worth to be studied and improved. In this paper, we will explore the influence of the dynamic camera motion blur and attempt to perform de-blurring on motion blurred image. During the experiment, GoPro is set on linear actuator to capture images at controlled movement speed to record motion images. According to capture motion images at different speeds, building the motion image de-blurring system based on the effectiveness parameters.
\end{abstract}

Keywords: motion blur, image reconstruction, Wiener filter, point spread function

\section{Introduction}

Multimedia image-related technology reaches a certain level, but there are still some challenges on image quality. It is worthy to be discussed and improved. Such as capturing fast-moving scenes or target, likely to cause blurred images, that is called motion blur. Many literatures are focusing on this problem and propose their own methods to improve it. Most of the core concept is try to find the blur kernel and uses a valid de-blurring method to reduce the blur area. Existing image processing technologies include restoration, enhancement, compression, and recognition. The blur in an image is caused by different reasons, including out-of-focus blur, atmospheric turbulence blur, motion blur, and electronic noise. In this paper, we concentrate our attention on image restoration for motion blur. According to the above-described image blur problem, this paper major focus on motion blur result of photography equipment movement too fast. The ultimate aim of this paper is to investigate motion blur image depends on its speed change factor. According different speed effective blur kernel parameters can be found. Some specific de-blurring methods can be used to restore blurred images. This paper is organized as follows. In Section 2, we briefly review some related work; in section 3, we introduce the de-blurring-based method in great detail; some experimental results are provided in Section 4; and Section 5 is the conclusion.

\section{Related Work}

In the past, there had been many researches for solving the problem of blurred images, including de-blur by using multi-image fusion [4], or de-blur by using a single image [2]. Amongst these, it is generally considered that the blur kernel is spatially uniform, which allows to be estimated from global image. Although some information is unknown, but most of the information is from the majority of the image can be estimated. Levin et al. [1] believed that the blur kernel is desired to be estimated and then used in restore the blur image. In this blur kernel is also be called the point spread function $(P S F)$ as shown in Fig. 1.

\footnotetext{
+ Corresponding author. Tel.: +886-4-23323000; fax: +886-4-23742337.

E-mail address: chlee@cyut.edu.tw.
} 


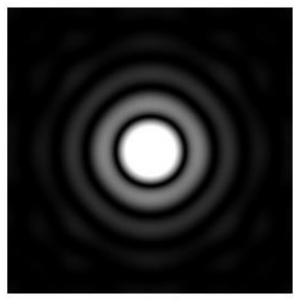

(a)

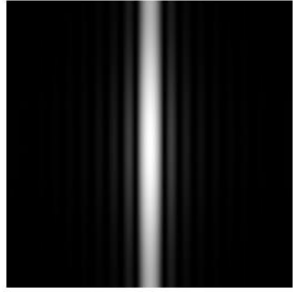

(b)

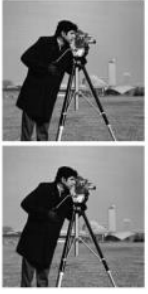

(a)

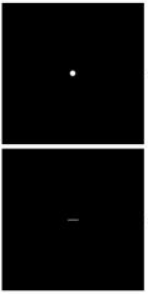

(b)

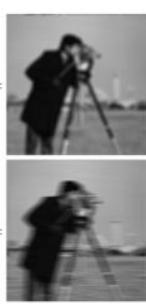

(c)

Fig. 1: The point spread function. (a) Out of focus blur.

(b) Motion blur.

Fig. 2: The degradation of image processing. (a) Original images. (b) Point spread function. (c) Blurred images.

Image de-convolution is based on blurred image (Fig. 2 (c)) and point spread function (Fig. 2(b)) to rebuild the original image (Fig. 2 (a)). This is well known that estimate the point spread function $(P S F)$ is an ill-posed problem. The original image convoluted by PSF to observe blurred image [2] [9], then the degradation of image processing as shown in Fig. 2.

The blur in an image is caused by different reasons, including out-of-focus blur, atmospheric turbulence blur, motion blur, and electronic noise. In view of the above problem of image blur, image restoration methods have been proposed in the past by many researches. Wen and Lee et al. [8] used an inverse filter and Wiener filter to restore blurred images in motion blur and out-of-focus blur. In our study, we use Wiener filter for image restoration because this method outperforms inverse filter in most cases.

\section{The Proposed Method}

In this paper, the GoPro camera is set up on linear actuators to capture images for motion blur, accordance with the case where different movement speed to estimate the approximate point spread function of motion blur. The blurred image by this estimated point spread function, and then used suggest de-blurring method with estimating PSF. The final image obtained will approximate the original image. The flow of the proposed image restoration process is shown in Fig. 3.

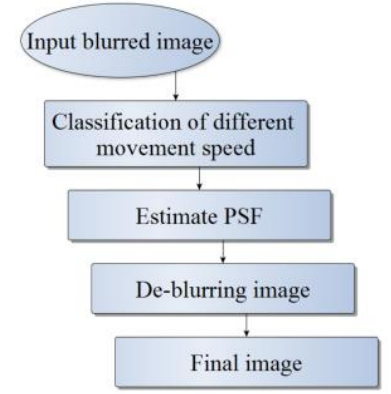

Fig. 3: The flow of the image restoration process.

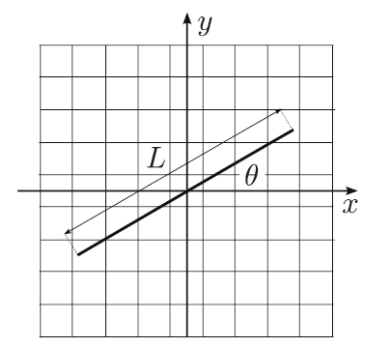

Fig. 4: Proposed discretized kernel for linear motion blur, length $L$ and angle $\theta$.

\subsection{The point spread function of motion blur}

We describe $P S F$ as $h(x, y)$, which is the $2 \mathrm{D}$ impulse response produced from a point source of light passing through the degradation system in the absence of noise. Although there have more the method to estimate the $P S F$ in the past, but in practical image restoration, it is not easy to obtain the exact parameters of $P S F$ [8]. Linear uniform motion blur generate from the linear movement of the entire image with along one direction. We assume that these movements are due to camera translation without in neither plane rotation nor changes of focus. We also assume that the whole scene is far away from the camera, thus the whole image is equally affected by the motion, yielding a spatially invariant motion blur [5].

The linear uniform motion blur PSF is a normalized delta function, supported on a line segment with length $L$ at an angle $\theta$ (Fig. 4)[5]. The angle $\theta$ depends on the motion direction, and the length $L$ is proportional to the motion speed and duration of exposure. This model corresponds to considering a bright spot moving along a straight line segment centered at the origin. Therefore, the linear motion blur PSF can be modeled as the following equation: 


$$
(x, y ; L, \theta)=\left\{\begin{array}{lc}
\frac{1}{L}, & \text { if } \sqrt{x^{2}+y^{2}} \leq \frac{L}{2} \text { and } \frac{x}{y}=-\tan \theta \\
0, & \text { elsewise }
\end{array}\right.
$$

\subsection{Image degradation model}

Denoting the original image as $f(x, y)$, where $x$ and $y$ are coordinates of a point on the image; the $P S F$ that blurs the original image as $h(x, y)$; an additive noise term as $n(x, y)$; and the blurred (or observed) image as $g(x, y)$, image degradation due to the ambiguity can be locally modeled as convolution [9], and the image degradation formula as follows:

$$
g(x, y)=h(x, y) * f(x, y)+n(x, y)
$$

where $*$ is the convolution operator. The equation (2) using the Fourier transforms can be derived in the frequency domain:

$$
G(u, v)=H(u, v) * F(u, v)+N(u, v)
$$

where $G(u, v), H(u, v), F(u, v)$ and $N(u, v)$ denotes the Fourier transform of $g(x, y), h(x, y), f(x, y)$ and $n(x, y)$, respectively. In optical theory, $H(u, v)$ is called the optical transfer function $(O T F)$. The goal of image restoration from blur image $G(u, v)$ and blur kernel $H(u, v)$ to estimate the original image $F(u, v)$. Based on the equation (3), we can estimate the original image by linear algebra methods. Based on the PSF of motion blur, we applied the Wiener filter and blind de-convolution to restore the blurred image.

\subsection{Wiener filter}

De-convolution image restoration process is to reduce or eliminate noise, but also mainly related to noise amplification. Wiener filter is a linear space-invariant filter, which makes use of the power spectrum of both the image and noise to prevent excessive noise amplification. Wiener de-convolution introduces a pseudoinverse filter in frequency domain, expressed as [8] [9]

$$
\hat{F}(u, v)=\left[\frac{H^{*}(u, v)}{|H(u, v)|^{2}+\frac{1}{S N R}}\right] G(u, v)
$$

where $S N R$ denotes the signal to noise ratio, helps to suppress high frequency part of the inverse filter. $H^{*}(u, v)$ is the complex conjugate of $H(u, v)$ in the high frequency domain.

$$
|H(u, v)|^{2}=H^{*}(u, v) H(u, v)
$$

Therefore, we can rewrite the equation (6) via equation (4), and when the $S N R$ information is unknown, we assume it is $K$ [8].

$$
\hat{F}(u, v)=\left[\frac{H^{2}(u, v)}{|H(u, v)|^{2}+K}\right] \frac{G(u, v)}{H(u, v)}
$$

where $K$ is the ratio of noise power spectrum to the original image power spectrum and is considered a constant. Wiener filtering is a more advanced regularization of inverse filtering; this filter displays better stability than pseudo inverse de-convolution. It has properties of a band-pass and is therefore even wellsuited to deal with moderate noise [7].

\subsection{Iterative blind deconvolution}

The iterative blind de-convolution is proposed by Ayers and Dainty is the most popular method, and requirements of the method that the PSF must be nonnegative with known finite support [3]. This approach is iterative between the space and Fourier domain, some constraints in the implementation of each step to improve the solution. The algorithm alternates between image and the Fourier domain, execution of each constraint conditions are known. The constraints are based on information available related the image and 
$P S F$. The Fourier domain constraint involves estimating the PSF by using the Fourier transform of the degraded image and estimated $P S F$. That is at the $k$-th iteration, and the equation is as follows [6]

$$
\begin{aligned}
& \hat{H}_{k}(u, v)=\left[\frac{G(u, v) F_{k-1}^{*}(u, v)}{\left|F_{k-1}(u, v)\right|^{2}+\alpha /\left|H_{k-1}(u, v)\right|^{2}}\right] \\
& \hat{F}_{k}(u, v)=\left[\frac{G(u, v) H_{k-1}^{*}(u, v)}{\left|H_{k-1}(u, v)\right|^{2}+\alpha /\left|F_{k-1}(u, v)\right|^{2}}\right]
\end{aligned}
$$

where $H^{*}{ }_{k-1}(u, v)$ and $F_{k-1}^{*}(u, v)$ denotes the complex conjugate of $H(u, v)$ and $F(u, v)$. The actual parameter $\alpha$ represents the energy of the additional noise and is determined by prior knowledge of the noise contamination level. If allowed that the $\alpha$ value must be carefully selected for a reliable restoration.

\section{Experimental Results}

\subsection{Motion blur image of different movement speed}

The GoPro camera is set up on linear actuators to capture motion blur images at different movement speed. The Cup is put on the table. Therefore, the left image in Fig. 5 shows that movement speed 10000 [Hz] of motion blur image, and the right image in Fig. 5 shows that movement speed $15000[\mathrm{~Hz}]$ of motion blur image. Accordance with different movement speeds to estimate the approximate point spread function of motion blur, in addition, due to the direction of movement of the horizontal movement, so that $P S F^{\prime} s$ angle $\theta=0$ is set. Finally, Wiener filter is used to restore motion blur image with estimate PSF. Experimental result and detail show in Fig. 6-9.
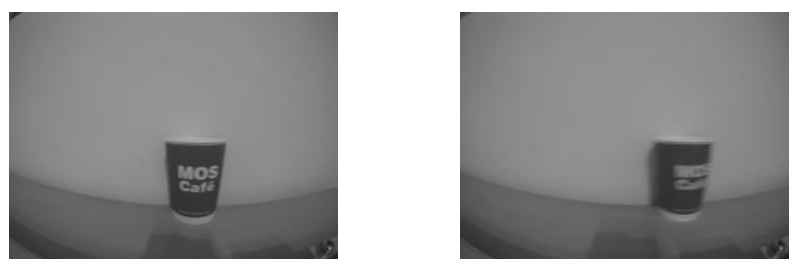

Fig. 5: The left image shows that motion blur image of movement speed 10000 [Hz]. Right image shows that motion blur image of movement speed $15000[\mathrm{~Hz}]$.

\subsection{Comparison with iterative blind deconvolution}

From Fig. 6 the left image shows that experimental results and detail of restored image at movement speed $10000[\mathrm{~Hz}]$ by using Wiener filter with PSF's parameter $L=11$ and $\theta=0$. Right image shows that experimental results of restored image at movement speed $10000[\mathrm{~Hz}]$ by using iterative blind deconvolution with same PSF. Fig. 7 zoom in on the cup.
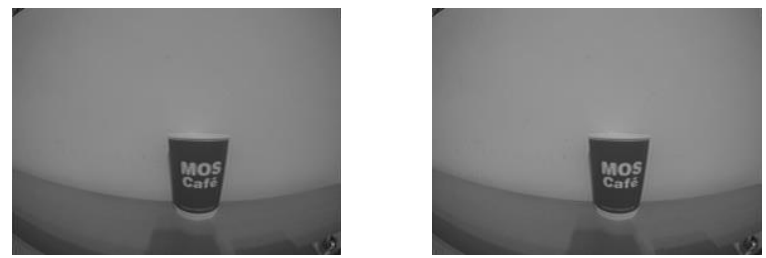

Fig. 6: The left image shows the result of using Wiener filter at movement speed $10000[\mathrm{~Hz}]$, right image show the result of using iterative blind deconvolution at movement speed $10000[\mathrm{~Hz}]$.
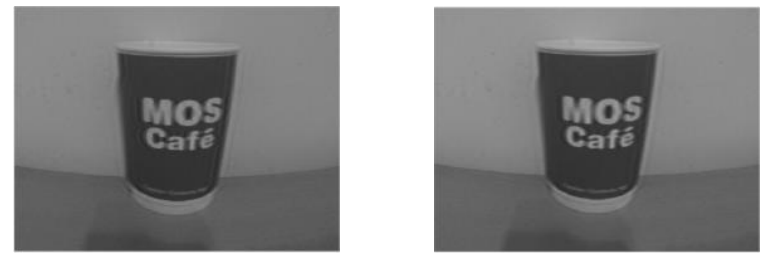

Fig. 7: The left image shows the detail of restored image using Wiener filter. Right image shows the detail of restored image using iterative blind deconvolution.

From Fig. 8 the left image show that experimental results and detail of restored image at movement speed $15000[\mathrm{~Hz}]$ by using Wiener filter with PSF's parameter $L=28$ and $\theta=0$. Right image show that experimental results of restored image at movement speed $15000[\mathrm{~Hz}]$ by using iterative blind deconvolution with same PSF. Fig.9 zoom in on the cup. 

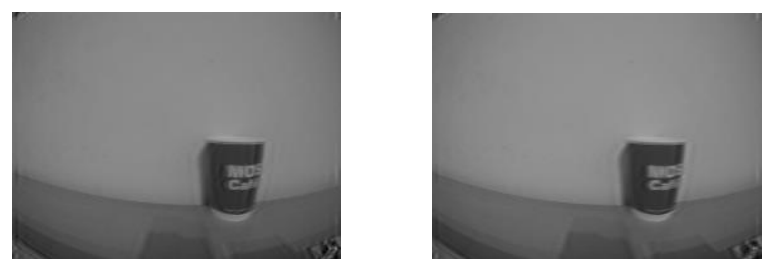

Fig. 8: The left image shows the result of using Wiener filter at movement speed $15000[\mathrm{~Hz}]$. Right image shows the result of using iterative blind deconvolution at movement speed $15000[\mathrm{~Hz}]$.
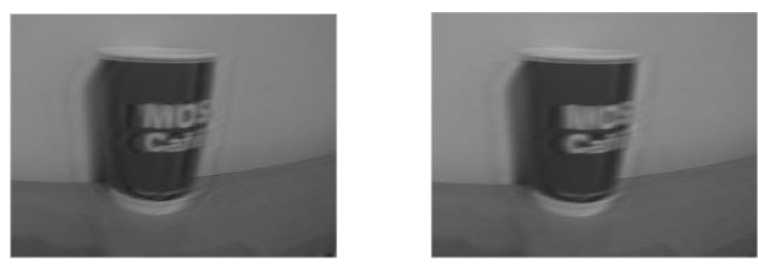

Fig. 9: The left image shows the detail of restored image using Wiener filter. Right image shows the detail of restored image using iterative blind deconvolution.

From Fig. 7 and 9 we can know that the detail of restored image by using Wiener performance is better than iterative blind deconvolution, because that obtained more detailed information on restoring image using Wiener filter.

\section{Conclusions}

In this paper the main goal was to investigate the use of GoPro camera on a linear actuator to capture motion image lead to motion blur. On the other hand, the relationship of movement speed and motion blur is analysis, so that we generated blur kernel with suitable Length $L$ and $\theta$ angle. Then de-blur image is conducted by using our proposed method. In addition, in this paper not only using Wiener filter to restore blurred image, but also using iterative blind deconvolution to experiment and compare performance. The experimental results show that the proposed Wiener filter to restore the image for motion blur performance is better than iterative blind deconvolution. We already proposed de-blurring system based on different speed for dynamic photography in this paper, existing good solution for de-blur effect of linear blur image. Therefore, in the future work we hope to be attached inertial measurement unit to collect data of the threeaxis acceleration, in order to explore the reasons of impact for motion blur between movement speed and acceleration.

\section{Acknowledgements}

This research was supported by the Ministry of Science and Technology of Taiwan under Grant MOST104-2221-E-324-025-

\section{References}

[1] A. Levin, Y. Weiss, F. Durand, and W. T. Freeman. Understanding and evaluating blind deconvolution algorithms. In: CVPR, 2009, pp. 1964-1971.

[2] F. Couzinie-Devy, J. Sun, K. Alahari, J. Ponce. Learning to estimate and remove non-uniform image blur. IEEE Conf., In: CVPR, 2013, pp. 1075-1082.

[3] G. R. Ayers and J. C. Dainty. Interative blind deconvolution method and its applications. Opt. Lett, 1988.

[4] H. A. Eltoukhy and S. Kavusi. A computationally efficient algorithm for multi-focus image reconstruction. In: Proc. SPIE Electron Imaging, 2003, Vol. 5017, pp. 332-341.

[5] J. Oliveira, M.A.T. Figueiredo, and J. Bioucas-Dias. Parametric blur estimation for blind restoration of natural images: linear motion and out-of-focus. IEEE Trans. Image Process., 2014, Vol. 23, pp. 466-477.

[6] D. Kundur, D. Hatzinakos. Blind image deconvolution. IEEE Signal Processing Magazine, 1996, 13 (3): pp. $43-$ 64.

[7] M. Welk, D. Theis, T. Brox, and J. Weickert. PDE-based deconvolution with forward-backward diffusivities and diffusion tensors. In: Proc. 5th Int. Conf. Scale Space and PDE Methods in Computer Vision, 2005, Vol. 3439, pp. $585-597$.

[8] C.Y. Wen, C.H. Lee. Point spread functions and their applications to forensic image restoration. Forensic Sci. J., 2002, Vol. 1, pp. 15-26.

[9] Yosuke Bando. Single-Shot Image Deblurring with Modified Camera Optics. PhD Thesis, The University of Tokyo, 2009. 